\title{
BMJ Open Determining the frequency of pathogenic germline variants from exome sequencing in patients with castrate-resistant prostate cancer
}

Steven N Hart, ${ }^{1,2}$ Marissa S Ellingson, ${ }^{1}$ Kim Schahl, ${ }^{1}$ Peter T Vedell, ${ }^{2}$ Rachel E Carlson, ${ }^{2}$ Jason P Sinnwell, ${ }^{2}$ Poulami Barman,, ${ }^{2}$ Hugues Sicotte, ${ }^{2}$ Jeanette E Eckel-Passow, ${ }^{2}$ Liguo Wang, ${ }^{1,2}$ Krishna R Kalari, ${ }^{1,2}$ Rui Qin, ${ }^{2}$ Teresa M Kruisselbrink, ${ }^{1}$ Rafael E Jimenez, ${ }^{3}$ Alan $\mathrm{H}$ Bryce, ${ }^{4}$ Winston Tan, ${ }^{5}$ Richard Weinshilboum, ${ }^{1,6}$ Liewei Wang, ${ }^{1,6}$ Manish Kohli ${ }^{7}$

To cite: Hart SN,

Ellingson MS, Schahl K, et al. Determining the frequency of pathogenic germline variants from exome sequencing in patients with castrateresistant prostate cancer. BMJ Open 2016;6: 010332. doi:10.1136/bmjopen-2015010332

- Prepublication history and additional material is available. To view please visit the journal (http://dx.doi.org/ 10.1136/bmjopen-2015010332).

LW and MK joint senior authors.

Received 21 October 2015 Revised 23 December 2015 Accepted 9 February 2016

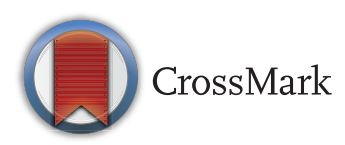

For numbered affiliations see end of article.

Correspondence to Dr Manish Kohli; kohli.manish@mayo.edu

\section{ABSTRACT}

Objectives: To determine the frequency of pathogenic inherited mutations in 157 select genes from patients with metastatic castrate-resistant prostate cancer (mCRPC).

Design: Observational.

Setting: Multisite US-based cohort.

Participants: Seventy-one adult male patients with histological confirmation of prostate cancer, and had progressive disease while on androgen deprivation therapy.

Results: Twelve patients (17.4\%) showed evidence of carrying pathogenic or likely pathogenic germline variants in the ATM, ATR, BRCA2, FANCL, MSR1, MUTYH, RB1, TSHR and WRN genes. All but one patient opted in to receive clinically actionable results at the time of study initiation. We also found that pathogenic germline BRCA2 variants appear to be enriched in $\mathrm{MCRPC}$ compared to familial prostate cancers.

Conclusions: Pathogenic variants in cancersusceptibility genes are frequently observed in patients with mCRPC. A substantial proportion of patients with mCRPC or their family members would derive clinical utility from mutation screening.

Trial registration number: NCT01953640; Results.

\section{INTRODUCTION}

Prostate cancer is the most prevalent cancer in men and is the second leading cause of cancer deaths in men. ${ }^{1}$ Localised prostate cancer (PC) has an excellent survival rate after 10 years. $^{2}$ However, $10-20 \%$ of men $^{3} 4$ will develop metastatic hormone-sensitive PC followed by metastatic castrate-resistant PC (mCRPC). The initial treatment for hormone-sensitive disease is hormonal ablation, which is successful in controlling

\section{Strengths and limitations of this study}

We describe the results of germline testing from a large cohort in the metastatic castrate-resistant prostate cancer setting, a population often overlooked for inherited mutation screening.

- These results have implications for the patient and their family members who may also be carrying the same genetic mutations.

- In lieu of a traditional replication set, we relied on previous estimates of mutation frequencies in this population which support our initial findings.

- Even though the entire exome was sequenced we only analysed data from 157 genes with known association with inherited cancer risk, so there may be other functionally relevant mutations not discussed here.

disease progression before castrate resistance emerges in the vast majority of patients. The median overall survival of mCRPC in 2004 was observed to be 18 months. ${ }^{4}$ Recently, progress has been made and this survival has doubled since 2004. ${ }^{5}$ While many factors contribute to the development of $\mathrm{PC}$ and progression to mCRPC, evidence suggests that pathogenic germline variants in known cancer predisposition genes such as BRCA2 can increase the risk of developing PC up to fivefold. ${ }^{6}$ Moreover, men with predisposing variants in BRCA2 present clinically with more aggressive stage and grade, ${ }^{7}$ leading to decreased survival compared with noncarriers. ${ }^{8}$ Beyond BRCA2, panel-based screening of 191 men with familial PC identified pathogenic truncating mutations in $A T M$, BRIP1, CHEK2, BRCA1, MUTYH, PALB2 and PMS2 in $7-27 \%$ of cases. ${ }^{9}$ After excluding 
$B R C A 2$, loss-of-function mutations were highly enriched in advanced disease, supporting the notion that multiple genes are involved in the hereditary pathogenesis of advanced PC.

Currently, it is not routinely recommended that patients diagnosed with PC have genetic testing, without some other mitigating factor such as a personal or family history of cancer, since the frequency of pathogenic $B R C A 2$ variants is low $(<1 \%)$ in unselected populations. ${ }^{10}$ Founder mutations, however, drive allele frequencies higher in Ashkenazi Jewish $(2.9 \%)^{11}$ and Icelandic populations $(5.6 \%) .^{7}$ Current National Comprehensive Cancer Network (NCCN) guidelines suggest screening for BRCA1 and BRCA2 mutations (also known as hereditary breast and ovarian cancer (HBOC) syndrome) if they present with a high Gleason score $(\geq 7)$ and there is a close relative with breast ( $\leq 50$ years), ovarian, pancreas or prostate cancer (also Gleason score z7) (http://www.nccn.org/).

In contrast to PC, very little is known about the prevalence of inherited pathogenic germline variants in patients who have progressed to mCRPC stage after initial treatments. To our knowledge, only one study has investigated the contribution of germline mutations in the mCRPC setting from comprehensive next-generation sequencing. ${ }^{12}$ In it, Robinson et al made the observation that $1 \%$ of mCRPC cases had germline mutations in $A T M$ and $6 \%$ carried mutations in BRCA2. No mention was made of inherited mutations in other known cancer predisposition genes.

In this study, we evaluated germline variants in men participating in the Prostate Cancer Medically Optimized Genome-Enhanced Therapy (PROMOTE) study at Mayo Clinic. The primary aim of PROMOTE is to identify molecular signatures of resistance and response to a standard hormonal therapy in mCRPC. Another objective, which is presented here, is to investigate the frequency of pathogenic germline variants in men with mCRPC and develop a protocol for return of clinically actionable results.

\section{METHODS}

\section{Patient eligibility}

Seventy-one male patients were selected if they were at least aged 18 years with histological confirmation of PC and had progressive disease while on androgen deprivation therapy (see table 1). Patients were recruited from three Mayo Clinic sites (Rochester MN, Jacksonville FL and Scottsdale AZ). All studies were conducted in accordance with the Institutional Review Board at Mayo Clinic and after obtaining patient consent.

\section{DNA sequencing analysis}

Genomic DNA was extracted from peripheral blood mononuclear cells using the QIAGEN FlexiGene chemistry on an AutoGen Flex Star. The red cells were lysed to allow the white cells to be pelleted by centrifugation.
Table 1 Descriptive statistics of the cohort

\begin{tabular}{|c|c|c|c|}
\hline & \multicolumn{2}{|c|}{$\begin{array}{l}\text { Deleterious } \\
\text { mutation }\end{array}$} & \multirow[b]{2}{*}{ p Value } \\
\hline & Yes & No & \\
\hline \multicolumn{4}{|l|}{ Age } \\
\hline At primary diagnosis & 64 & 66 & 0.047 \\
\hline At ADT & 69.4 & 69.5 & 0.958 \\
\hline $\begin{array}{l}\text { At the development } \\
\text { of } \mathrm{mCRPC}\end{array}$ & 73.9 & 73.7 & 0.923 \\
\hline \multicolumn{4}{|l|}{ Family history } \\
\hline Yes & 10 & 45 & $0.785^{\star}$ \\
\hline No & 3 & 11 & \\
\hline \multicolumn{4}{|l|}{ Histology } \\
\hline Adenocarcinoma & 5 & 28 & $0.877 \dagger$ \\
\hline $\begin{array}{l}\text { Poorly differentiated } \\
\text { carcinoma NOS }\end{array}$ & 1 & 8 & \\
\hline Small-cell carcinoma & 0 & 1 & \\
\hline Unknown & 7 & 20 & \\
\hline \multicolumn{4}{|l|}{ Gleason score } \\
\hline 7 & 0 & 2 & $0.054^{*}$ \\
\hline 8 & 0 & 15 & \\
\hline 9 & 2 & 6 & \\
\hline 10 & 4 & 13 & \\
\hline NS & 7 & 21 & \\
\hline \multicolumn{4}{|c|}{$\begin{array}{l}\text { *Linear model ANOVA. } \\
\text { †Pearson's } \chi^{2} \text { test. } \\
\text { ADT, androgen deprivation therapy; mCRPC, metastatic } \\
\text { castrate-resistant prostate cancer; NS, not scored. }\end{array}$} \\
\hline
\end{tabular}

Pellets were lysed and treated with protease to remove proteins from the sample. DNA was precipitated out with isopropanol and washed with $70 \%$ ethanol. DNA was then resuspended in Tris-EDTA (TE) buffer and quantified using a spectrophotometer.

Paired-end libraries were prepared following the manufacturer's protocol (Agilent) using the Bravo liquid handler (Agilent). The concentration and size distribution of the libraries was determined on an Agilent Bioanalyzer DNA 1000 chip. Whole exome capture was performed using a modified Agilent V4+UTR kit, which spans $71.45 \mathrm{Mb}$ of coding exons, plus an intronic region tiling of the androgen receptor gene. Concentration and size distribution of the libraries was determined on Qubit (Invitrogen) and Agilent Bioanalyzer DNA 1000 chip for quality control purposes. Exome libraries were loaded one sample per lane onto Illumina TruSeq v3 paired-end flow cells at concentrations of $9 \mathrm{pM}$ following Illumina's standard protocol using the Illumina cBot and TruSeq rapid paired-end cluster kit V.3.

On average, 200-300 million $100 \mathrm{bp}$ paired-end reads were sequenced per sample. A sample passed initial quality control if more than $90 \%$ of the capture region was covered at a minimum of $\times 20$ and more than $70 \%$ of the capture kit covered at $\times 50$. Unless otherwise specified, all bioinformatics tools were run under default configuration. Reads were aligned to the hg19 reference genome using Novoalign (http://novocraft.com; VN: V2.07.13) with the following options: -hdrhd off -v 120 
-c 4 -i PE 425,80 -x 5 -r Random. Realignment and recalibration was performed using GATK (VN: 2.7-4-g6f46d11) Best Practices V.3. Germline variations were called with GATK's UnifiedGenotyper.

\section{Variant annotation and prioritisation}

SAVANT (VN:1.1.0) was used to annotate the functional impact of variants. Using the BioR toolkit, ${ }^{13}$ several annotation sources were also added to aid in filtering/ prioritising genetic variants. Common variants were excluded if they were seen at more than $1 \%$ allele frequency in the Exome Sequencing Project (ESP) database. ${ }^{14}$ Variants were then further restricted to a subset of 157 target genes (see online supplementary table S1) associated with hereditary cancer risk. Any variant that was reported as a 'disease-causing mutation' in the Human Gene Mutation Database (HGMD) ${ }^{15}$ reported as pathogenic in $\mathrm{ClinVar}^{16}$ or was predicted to be protein truncating (frameshift, nonsense or splice site variants) underwent comprehensive review by a certified genetic counsellor. This included review of allele frequency data, variant databases, literature and in silico predictions. Each variant was classified as benign, likely benign, likely pathogenic, pathogenic or unknown significance in accordance with American College of Medical Genetics (ACMG) guidelines. ${ }^{17}$

\section{Return of results}

Return of results was offered to patients with a pathogenic or likely pathogenic variant in one of the genes that was determined to be medically actionable, which was defined as having existing medical management guidelines. Return of results was not considered for childhood-onset conditions, autosomal recessive conditions (as it was not possible to determine whether two variants identified within a gene were in cis or in trans) or carrier status for autosomal recessive conditions. Patients were eligible for return of results if they were not already aware of the result through clinical testing and if they had opted in to receive results on the consent form at the time of enrolment.

Patients eligible for return of results were contacted by a study coordinator and offered a telephone or in-person consultation with a certified genetic counsellor for discussion of risks, benefits and limitations of receiving germline research results. A second consultation with a certified genetic counsellor was then offered for results disclosure. Given that the results were generated in a research laboratory, if patients had elected to receive results, confirmatory testing in a Clinical Laboratory Improvement Amendments (CLIA)-certified laboratory would have been recommended prior to using the results to make medical management decisions.

\section{RESULTS}

Between June 2013 and August 2014, 71 patients with mCRPC were recruited in the study. Germline variant analysis was completed on 69 patients. Table 1 highlights the demographics of the study cohort. No significant associations were found between having a pathogenic or likely pathogenic variant and the age at primary disease, age at androgen insensitivity or metastasis. There was also no association between having a pathogenic or likely pathogenic variant and time to progression between primary diagnosis and castrate resistance ( $p=0.26$, Student's t test) or between castrate resistance and metastasis ( $p=0.83$, Student's $t$ test). We did not observe any significant associations related to the histology and pathogenic variant status, or family history (defined as other first-degree relative with cancer). One sample was identified as adenocarcinoma but did not have enough tumour $(<5 \%)$ for identifying Gleason score (table 2).

In all, 12 pathogenic or likely pathogenic variants were identified in nine genes: $A T M, A T R, B R C A 2$, FANCL, MSR1, MUTYH, RB1, TSHR and WRN. ATM and $B R C A 2$ were the only genes mutated in more than one patient. All of the pathogenic variants identified were previously labelled as pathogenic/disease causing by either ClinVar or HGMD, except for the ATR, MSR1, $R B 1$ and $W R N$ variants. None of these variants have been shown to be directly linked to mCRPC but are markedly enriched for other cancer types.

\section{Return of results}

At the initiation of the study, 68/69 participants opted in to receive clinically actionable germline results on the consent form at the time of enrolment. Of the 12 pathogenic or likely pathogenic variants, only three were considered medically actionable and met the criteria for return of results (all in BRCA2). These patients were recontacted by a study coordinator to offer a consultation with a genetic counsellor for results disclosure. One patient changed his mind and verbally opted out of knowing his results. The second patient proceeded with an initial consultation with a genetic counsellor and planned to discuss the information with his family prior to deciding whether or not to proceed with results disclosure. The third patient's daughter called to ask questions about what the genetic counselling appointment entailed. After speaking with the genetic counsellor for more information, she indicated her interest in proceeding with a genetic consultation with her father. After 10 months, neither of these two patients returned for results disclosure.

A family history of cancer was documented in the medical records of all three patients. The first patient reported a brother who died of $\mathrm{PC}$, six female relatives with postmenopausal breast cancer and a niece with uterine cancer. There was report of a 'cancer gene' in the family, but further details were not available. The second patient reported 'a history of pancreas cancer' in his family, but further details were not available. Finally, the third patient reported that his mother, brother and son had been diagnosed with colon cancer, a son had 
Table 2 Pathogenic and likely pathogenic germline variants in patients with $\mathrm{mCRPC}$

\begin{tabular}{|c|c|c|c|c|c|c|c|}
\hline Gene & Transcript & Variant & $\begin{array}{l}\text { Protein } \\
\text { effect }\end{array}$ & $\begin{array}{l}\text { PROMOTE } \\
\text { classification }\end{array}$ & $\begin{array}{l}\text { Pathogenic } \\
\text { in ClinVar }\end{array}$ & $\begin{array}{l}\text { Disease } \\
\text { causing } \\
\text { in HGMD }\end{array}$ & Phenotype \\
\hline ATM & NM_000051.3 & c.7271T>G; p.Val2424Gly & Missense & Pathogenic & Yes & Yes & Ataxia telangiectasia \\
\hline ATM & NM_000051.3 & $\begin{array}{l}\text { c.3245_3247delinsTGAT; } \\
\text { p.His1082LeufsX14 }\end{array}$ & Frameshift & Pathogenic & Yes & Yes & Ataxia telangiectasia \\
\hline ATR & NM_001184.3 & c.4957C>T; p.Arg1653X & Stop gained & Likely pathogenic & & & $\begin{array}{l}\text { Familial cutaneous telangiectasia and } \\
\text { cancer syndrome }\end{array}$ \\
\hline$B R C A 2$ & NM_000059.3 & $\begin{array}{l}\text { c.469_470delAA; } \\
\text { p.Lys157ValfsX25 }\end{array}$ & Frameshift & Likely pathogenic & Yes & Yes & $\begin{array}{l}\text { Hereditary breast/ovarian cancer } \\
\text { syndrome }\end{array}$ \\
\hline$B R C A 2$ & NM_000059.3 & $\begin{array}{l}\text { c.6444dupT; } p . \\
\text { Ile2149TyrfsX2 }\end{array}$ & Frameshift & Pathogenic & Yes & Yes & $\begin{array}{l}\text { Hereditary breast/ovarian cancer } \\
\text { syndrome }\end{array}$ \\
\hline BRCA2 & NM_000059.3 & $\begin{array}{l}\text { c.9513_9516delACTT; } \\
\text { p.Leu3172AlafsX44 }\end{array}$ & Frameshift & Pathogenic & Yes & & $\begin{array}{l}\text { Hereditary breast/ovarian cancer } \\
\text { syndrome }\end{array}$ \\
\hline FANCL & NM_001114636.1 & $\begin{array}{l}\text { c.1111_1114dupATTA; } \\
\text { p.Thr372AsnfsX13 }\end{array}$ & Frameshift & Likely pathogenic & & Yes & Fanconi anaemia \\
\hline MSR1 & NM_138715.2 & $\begin{array}{l}\text { c.183delT; } p . \\
\text { Phe61Leufs X10 }\end{array}$ & Frameshift & Likely pathogenic & & & Hereditary prostate cancer \\
\hline MUTYH & NM_001128425.1 & c.1187G>A; p.Gly396Asp & Missense & Pathogenic & Yes & Yes & MUTYH-associated polyposis \\
\hline$R B 1$ & NM_000321.2 & c. $1960+1 \mathrm{G}>\mathrm{C}$ & Splice donor & Likely pathogenic & & & retinoblastoma \\
\hline TSHR & NM_000369.2 & c. $1349 \mathrm{G}>\mathrm{A} ; \mathrm{p} . \mathrm{Arg} 450 \mathrm{His}$ & Missense & Pathogenic & & Yes & Thyroid carcinoma \\
\hline WRN & NM_000553.4 & c.525delG; p.Trp175X & Frameshift & Likely pathogenic & & & Werner syndrome \\
\hline
\end{tabular}


bone cancer and a sister had pancreatic cancer. While all three patients would likely have fulfilled NCCN criteria for HBOC testing, there was no documentation of referrals to medical genetics, consultations with medical genetics or genetic testing in the patients' medical records.

\section{DISCUSSION}

Here we report our findings of inherited pathogenic mutations in known cancer predisposition genes from the exome sequences of 69 mCRPC cases. We observed a high frequency of pathogenic germline variants in patients with mCRPC $(17.4 \% ; 12 / 69)$. Our finding is consistent with a recent report in which germline mutations in BRCA2 were found in $5.3 \%$ of 150 mCRPC cases. $^{12}$ Mutation frequencies reported here are also similar to those reported for ATM $(2 / 69,2.9 \%$ vs $2 / 150$, $1.3 \%)$ and BRCA1 $(0 / 69,0 \%$ vs $1 / 150,0.6 \%)$ genes, although our small sample size prevents a robust estimate of true allele frequencies in this population.

Clear links have established BRCA2 as a predisposition gene for PC. However, the frequency of pathogenic variants in BRCA2 is low-on the order of $0.5-1 \%$ in unselected populations. ${ }^{10}$ When we compare the frequency of pathogenic BRCA2 variants with PC from non-mCRPC patients, the enrichment is striking. The pathogenic BRCA2 variant frequency $(4.2 \%)$ in patients with mCRPC is much higher than has been reported in the context of familial PC. In a large study of 266 familial PC cases, pathogenic BRCA 2 variants were not observed $(\mathrm{p}<0.01$, Fisher's exact test), although smaller studies ( $<22$ families) have observed mutation frequencies near $5 \% .{ }^{18}{ }^{19}$ We also observed a higher frequency of pathogenic $B R C A 2$ variants in patients with mCRPC compared with early-onset PC cases $(p<0.06$, Fisher's exact test). In a study of 1589 men diagnosed with PC under age 65 , only $1.2 \%$ were found to have pathogenic BRCA2 mutations. ${ }^{20}$

\section{Other genes}

Excluding the ATM and BRCA2 genes, MUTYH is the only other gene that has previously been identified in these cohorts. Leongamornlert et at found one stopgain mutation in 191 individuals with familial PC, with an enrichment in metastatic cases. MUTYH mutation carriers are not thought to be at increased risk for PC, at least in the case of individuals with a family history of colorectal cancer. ${ }^{21}$ Even less is known about the role of ATR, FANCL, MSR1, MUTYH, RB1, TSHR and WRN in PC or MCRPC.

Finally, we note that the three patients with clinically actionable BRCA2 mutations would likely have met NCCN criteria for HBOC screening but did not undergo medical genetic consultations and/or testing previously. It is unknown if genetic screening and counselling is being offered in clinical practice as often as recommended by national guidelines to families with a history of multiple cancers. While interesting, the small sample size precludes us from determining whether or not this is significant; thus, more studies in this area are warranted.

\section{Author affiliations}

${ }^{1}$ Center for Individualized Medicine, Mayo Clinic, Rochester, Minnesota, USA

${ }^{2}$ Health Sciences Research, Mayo Clinic, Rochester, Minnesota, USA

${ }^{3}$ Department of Laboratory Medicine and Pathology, Mayo Clinic, Rochester, Minnesota, USA

${ }^{4}$ Division of Hematology/Oncology, Mayo Clinic, Mayo Clinic Cancer Center, Scottsdale, Arizona, USA

${ }^{5}$ Division of Hematology and Oncology, Department of Medicine, Mayo Clinic, Jacksonville, Florida, USA

${ }^{6}$ Department of Molecular Pharmacology \& Experimental Therapeutics, Mayo Clinic, Rochester, Minnesota, USA

${ }^{7}$ Division of Medical Oncology, Department of Oncology, Mayo Clinic, Rochester, USA

Twitter Follow Steven Hart at @StevenNHart

Acknowledgements The PROMOTE study is funded in part by the Mayo Clinic Center for Individualized Medicine; A.T. Suharya and Ghan D.H.; Joseph and Gail Gassner; and Mayo Clinic Schulze Cancer for Novel Therapeutics in Cancer Research. Other contributing groups include the Mayo Clinic Cancer Center and the Pharmacogenomics Research Network (PGRN).

Contributors MSE, TMK and KS classified and reviewed variants. SNH, PTV, HS, LW and KRK were responsible for the DNA sequencing and informatics. REC, JPS, PB, JEE-P and RQ coordinated the statistical analysis. WT, MK and AHB led the patient recruitment effort. The entire study was supervised and directed by RW, LW and MK. SNH, MSE and MK wrote the paper.

Funding Mayo Clinic Cancer Center (P30CA 15083-41). Joseph and Gail Gassner. Mayo Clinic Schulze Cancer for Novel Therapeutics in Cancer Research. Pharmacogenomics Research Network. A. T. Suharya and Ghan D. H. Mayo Clinic Center for Individualized Medicine.

Competing interests None declared.

Ethics approval Institutional Review Board.

Provenance and peer review Not commissioned; externally peer reviewed.

Data sharing statement No additional data are available.

Open Access This is an Open Access article distributed in accordance with the Creative Commons Attribution Non Commercial (CC BY-NC 4.0) license, which permits others to distribute, remix, adapt, build upon this work noncommercially, and license their derivative works on different terms, provided the original work is properly cited and the use is non-commercial. See: http:// creativecommons.org/licenses/by-nc/4.0/

\section{REFERENCES}

1. Siegel R, Naishadham D, Jemal A. Cancer statistics, 2013. CA Cancer J Clin 2013;63:11-30.

2. Klotz L, Zhang L, Lam A, et al. Clinical results of long-term follow-up of a large, active surveillance cohort with localized prostate cancer. $J$ Clin Oncol 2010;28:126-31.

3. Spratt DE, Zumsteg ZS, Pei X, et al. Predictors of castrationresistant prostate cancer after dose-escalated external beam radiotherapy. Prostate 2015;75:175-82.

4. Kirby M, Hirst C, Crawford ED. Characterising the castrationresistant prostate cancer population: a systematic review. Int J Clin Pract 2011;65:1180-92.

5. Ryan CJ, Smith MR, Fizazi K, et al. Abiraterone acetate plus prednisone versus placebo plus prednisone in chemotherapy-naive men with metastatic castration-resistant prostate cancer (COU-AA-302): final overall survival analysis of a randomised, double-blind, placebo-controlled phase 3 study. Lancet Oncol 2015;16:152-60.

6. Liede A, Karlan BY, Narod SA. Cancer risks for Male carriers of germline mutations in BRCA1 or BRCA2: a review of the literature. J Clin Oncol 2004;22:735-42.

7. Tryggvadottir L, Vidarsdottir L, Thorgeirsson $\mathrm{T}$, et al. Prostate cancer progression and survival in BRCA2 mutation carriers. J Natl Cancer Inst 2007;99:929-35. 
8. Narod SA, Neuhausen S, Vichodez G, et al. Rapid progression of prostate cancer in men with a BRCA2 mutation. Br J Cancer 2008;99:371-4

9. Leongamornlert D, Saunders E, Dadaev T, et al. Frequent germline deleterious mutations in DNA repair genes in familial prostate cancer cases are associated with advanced disease. $\mathrm{Br} J$ Cancer 2014:110:1663-72

10. Agalliu I, Karlins E, Kwon EM, et al. Rare germline mutations in the BRCA2 gene are associated with early-onset prostate cancer. $\mathrm{Br} \mathrm{J}$ Cancer 2007:97:826-31.

11. Agalliu I, Gern R, Leanza S, et al. Associations of high-grade prostate cancer with BRCA1 and BRCA2 founder mutations. Clin Cancer Res 2009;15:1112-20.

12. Robinson D, Van Allen EM, Wu YM, et al. Integrative clinical genomics of advanced prostate cancer. Cell 2015;161:1215-28.

13. Kocher JP, Quest DJ, Duffy P, et al. The Biological Reference Repository (BioR): a rapid and flexible system for genomics annotation. Bioinformatics 2014;30:1920-2.

14. Wang K, Li M, Hakonarson H. ANNOVAR: functional annotation of genetic variants from high-throughput sequencing data. Nucleic Acids Res 2010;38:e164.
15. Stenson PD, Ball EV, Mort M, et al. Human Gene Mutation Database (HGMD): 2003 update. Hum Mutat 2003;21:577-81.

16. Landrum MJ, Lee JM, Riley GR, et al. ClinVar: public archive of relationships among sequence variation and human phenotype. Nucleic Acids Res 2014;42(Database issue):D980-5.

17. Richards CS, Bale S, Bellissimo DB, et al. ACMG recommendations for standards for interpretation and reporting of sequence variations: revisions 2007. Genet Med 2008;10:294-300.

18. Sinclair CS, Berry R, Schaid D, et al. BRCA1 and BRCA2 have a limited role in familial prostate cancer. Cancer Res 2000;60:1371-5.

19. Agalliu I, Kwon EM, Zadory D, et al. Germline mutations in the BRCA2 gene and susceptibility to hereditary prostate cancer. Clin Cancer Res 2007;13:839-43.

20. Kote-Jarai Z, Leongamornlert D, Saunders E, et al. BRCA2 is a moderate penetrance gene contributing to young-onset prostate cancer: implications for genetic testing in prostate cancer patients. Br J Cancer 2011;105:1230-4.

21. Win AK, Cleary SP, Dowty JG, et al. Cancer risks for monoallelic MUTYH mutation carriers with a family history of colorectal cancer. Int J Cancer 2011;129:2256-62. 